\title{
DISPLACEMENT OF THE HEART IN PNEUMONIA IN CHILDHOOD.
}

BY

\author{
KENNETH H. TALLERMAN, M.D., M.R.C.P., \\ and MONTAGUE H. JUPE, D.M.R.E.
}

(From the Children's and Radiological Departments of the London Hospital.)

In cases of collapse of the lung it is well-known that the heart deviates towards the side of the lesion, and, since its description by Pasteur in 1890, this displacement of the heart has been generally regarded as the principal physical sign of the condition. That a similar phenomenon frequently occurs during the course of pneumonia in childhood is, however, not widely known, if one may judge by the scanty literature on the subject and the absence of references to it in textbooks. On this account it would seem to be of interest to report the following cases in which this condition was observed, and to deal in some detail with the subject.

Three of the cases mentioned below have already been shown, and their condition two years ago, briefly reported in the Proceedings of the Royal Society of Medicine ${ }^{1}$.

In 1922 Thoenes ${ }^{2}$ reported a series of 11 cases of pneumonia in infants, in all of which he noted displacement of the mediastinal contents, and especially of the heart, towards the affected lung. As the pneumonia resolved, the heart went back, though slowly, to its normal position.

The next paper to appear on this subject was that of Wallgren ${ }^{3}$, who reported 8 cases occurring both in infants and in older children, in which similar findings were noted. There appears to be no further publication in this connection until 1927, when Griffith ${ }^{4}$ reported displacement of the heart taking place during the course of pneumonia in 16 out of 40 cases, and gave details of the cases of 7 infants in which this occurred. It is difficult to be certain of the type of pneumonia present in the cases reported, since the symptoms, physical signs, and course of the disease are not all clearly defined. In Thoenes's series, however, lobar pneumonia and broncho-pneumonia would appear to have occurred in approximately equal numbers, while in Wallgren's and Griffith's cases, lobar pneumonia predominated. Griffith in fact states definitely that the majority of his cases conformed to the type of croupous pneumonia without discoverable signs of atelectasis.

The question arises as to the causation of this cardiac displacement, and it is this which is probably of chief interest. Two factors are apparently at work: on the one hand a traction of the mediastinal contents towards the affected side as the result of the shrinkage and partial collapse of the diseased lung; and on the other hand, a push exerted from the sound side by the unaffected lung, distended by compensatory emphysema. In postmortem examinations of cases dying of broncho-pneumonia, it is usual to find in the affected portions of lung, areas of collapse coexistent with areas of consolidation. Such a condition occurring in mass in the affected lung, 
together with emphysema-probably compensatory-on the opposite side, would seem to explain adequately both the deviation of the heart and the direction of its displacement. Wallgren and Griffith regard emphysema and distention of the sound lung as the chief factors concerned. Thoenes lays stress on collapse of the affected lung, and gives two autopsy records which support these views.

Coryllos and Birnbaum ${ }^{5}$ have thrown further light on this subject, and in their interesting paper recently published, bring forward evidence showing that pneumonia is an atelectasis associated with infection. Collapse of the lung, either of a whole lobe, or, in the case of broncho-pneumonia, a patchy atelectasis, would in their opinion be the cause of displacement of the mediastinal contents.

From a consideration of our five cases detailed below, together with those already referred to in the literature, it appears that in all probability both factors come into play in the causation of this phenomenon.

\section{Clinical Reports.}

Case I. M.G., age 1 month. Admitted to Hospital with typical symptoms of pneumonia. History of two days' cough, fever and embarrassed respiration.

$23 / 2 / 28$. (repitations and rhonchi heard over both lungs.

$3 / 3 / 28$. Slightly diminished air entry and impaired percussion note, right lower lobe.

6/3/28. X-ray photograph shows the heart and trachea both lying to the right, with a definite increase in density of the right upper lobe (Fig. 1).

$8 / 3 / 28$. Impaired percussion note and diminished breath sounds in right upper lobe, with crepitations throughout right lung. Area of cardiac dullness not defined. Apex beat appears to lie below the 4th space beneath the sternum.

13/3/28. X-ray photograph shows upper lobe of right lung clearing, with the heart slightly more normal in position, but still well to the right.

$30 / 3 / 28$. No definite physical signs now in chest, and heart appears in normal position on clinical examination. X-ray picture taken at this time shows heart and trachea returning towards the left, and the apex of right lung clear. ('hild's general condition good.

6/6/28. ('hild doing well and gaining weight satisfactorily. No abnormal physical signs. $\mathrm{X}$-ray shows heart practically normal in position ; slight degree of mottling at apex.

Case II. E.A., age 4 months. Said to have had bronchitis for three wecks. Three days before admission had a convulsion, and on the day of admission a further convulsion with feverishness and embarrassed respiration.

$2 / 2 / 28$. On admission respiration rapid; rhonchi heard throughout the left lung with harsh breath sounds. Over right lung, breath sounds high pitched, percussion note impaired, and crepitations and rhonchi throughout all lobes : the area of cardiac dullness cannot be defined, since there appears to be some emphysema over left side of chest, but the heart sounds are heard rather better to the right of the sternum. Spleen palpable.

$27 / 2 / 28$. X-ray photograph shows heart and trachea displaced to the right with areas of opacity in the middle and lower lobes of the right lung.

$1 / 3 / 28$. Respiration becoming normal. Left lung clear except for a few crepitations at the base. In the right lung physical signs remain the same, although the percussion note posteriorly is now normal. Heart sounds heard more clearly to the left than previously.

5/3/28. X-ray shows heart and trachea in approximately the same position, though the lung itself is clearing. The chief area of opacity is well seen on the screen, but is not so obvious in the X-ray film, being overlapped by the heart shadow.

20/3/28. Percussion note impaired and crepitations present at right base. Heart still appears to the right. X-ray picture shows lung clear and heart returning, although not yet in normal position. 
27/3/28. Crepitations still present at the right base. Clinically heart appears to be normar in position now.

17/4/28. Child seems very well. Physical signs at right base unchanged. X-ray picture shows right lung clear, and the heart and trachea now practically normal in position.

22/5/28. Some cough and bronchitis present. Crepitations also heard at right base.

5/6/28. Occasional crepitations right base; no other physical signs. No cough now, and child seems well. X-ray shows the lungs to be clear, but neither heart nor trachea has returned completely to its normal position.

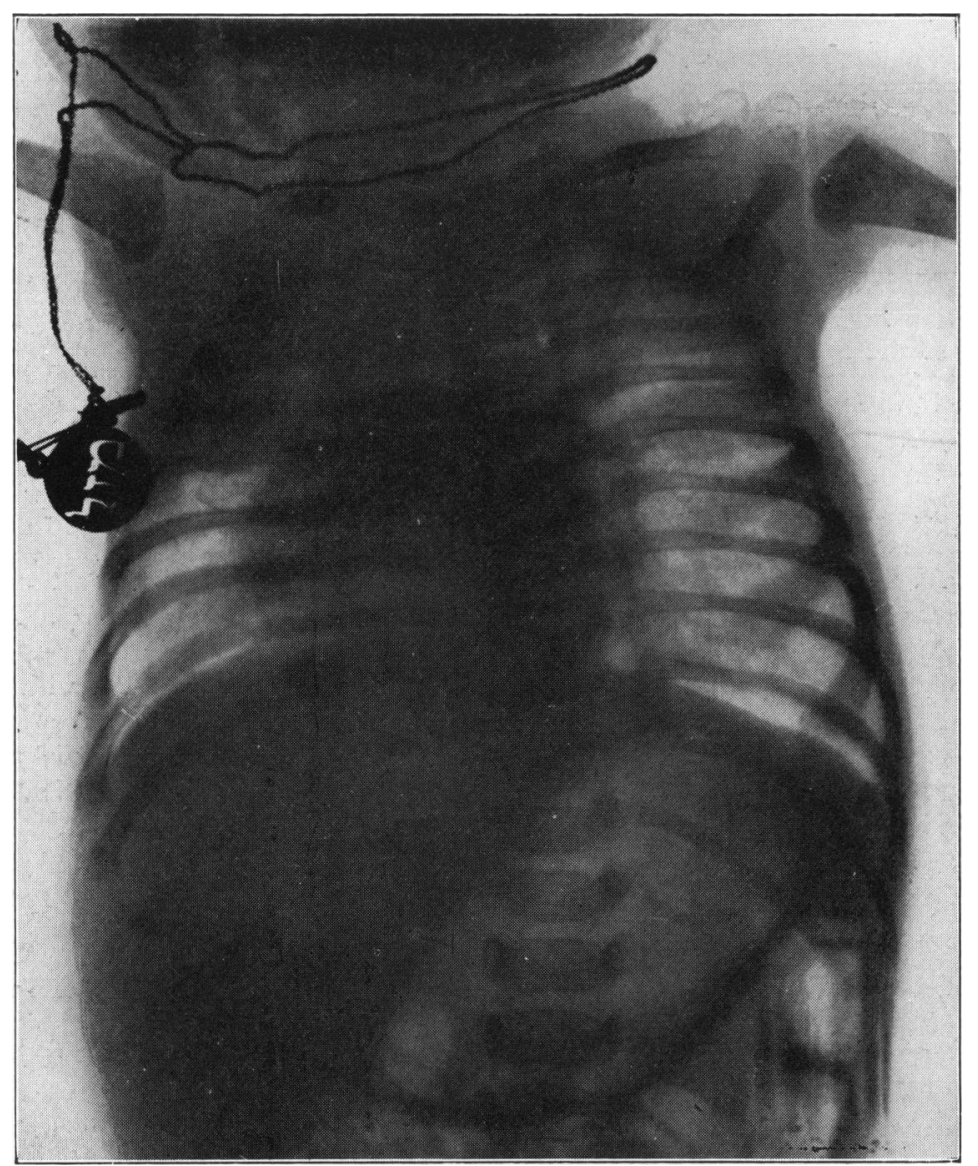

FIG. 1. Case I., M.G. $6 / 3 / 28$. The heart is seen to lie almcst entirely in the right side of the chest; the apex of the right lung is opaque.

$3 / 7 / 28$. Child well and gaining weight very satisfactorily. ('hest clear of physical signs.

18/8/28. Recurrence of cough. Rhonchi throughout both lungs, and some crepitations heard at the left base.

25/9/28. No cough now. Infant seems well though the chest is said to be "rattley " at times. She has cut no teeth yet, though her weight is up to normal. Fxcepting a few scattered rhonchi, there are no physical signs in the chest. X-ray shows that there is still a small area of increased density in the right lower lobe to right of heart. Heart appears normal in position now.

22/11/28. Crawling and starting to walk. Still no teeth (14 months old now). Apart from diminished breath sounds and a few crepitations heard over the right lower lobe on deep breathing, there is nothing abnormal to be noted in the chest now. 
Case III. L.M., age 10 months. Three days' history of vomiting and off food.

10/10/27. On admission, infant very cyanosed, with embarrassed respiration and marked stridor. Apparently much mucus in the trachea : throat swab negative. Coarse crepitations at both bases and rhonchi throughout both lungs. Heart apparently normal in position. Spleen palpable.

25/10/27. Dullness and diminished breath sounds right base. No area of cardiac dullness defined. Heart sounds heard most loudly just medial to right nipple.

26/10/27. X-ray photograph shows no cardiac shadow in left side of the chest, but an opacity throughout right side, with the trachea pulled over to the right. The picture was taken under difficulties, oxygen being administered by nasal catheter. (The question of a foreign body in the bronchus was considered at this time.)

28/10/27. No change radiologically (Fig. 2).

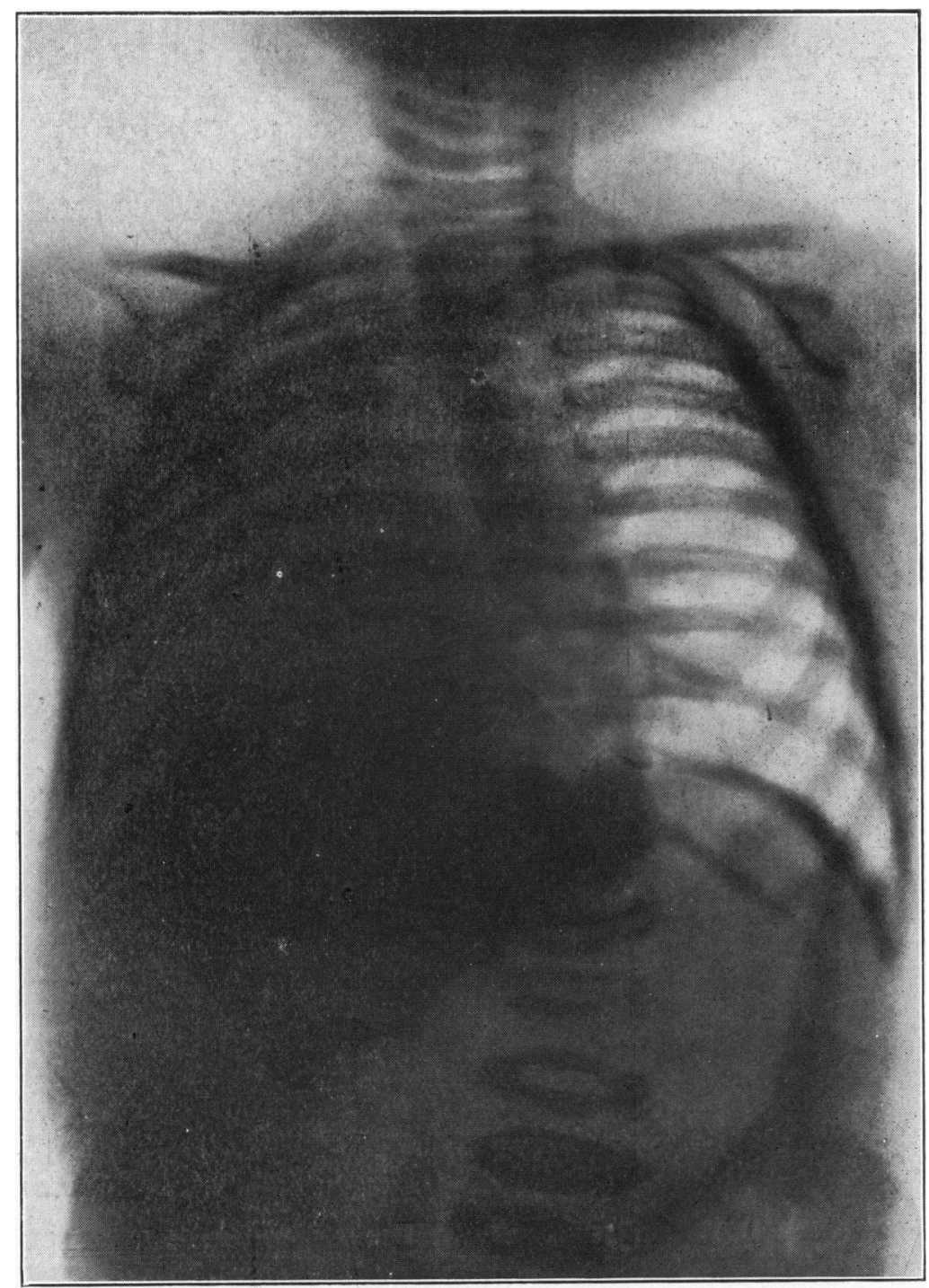

Fig. 2. Case III., L.M. 28/10/27. The heart lies completely in the right side of the chest, which is opaque, so that no cardiac sliadow is seen. 'Lhe trachea is seen well to the right of the mid-line. 
$31 / 10 / 27$. Dullness, bronchial breath sounds and rhonchi throughout right lung, with deficient movement of right side of chest.

3/11/27. Percussion note still impaired. Breath sounds high pitched over right lower and middle lobes. Hcant displaced to right and respiration rate still raised, but temperature now normal.

From now onwards the right lung cleared, and the heart gradually returned to the normal position as shown by physical signs, and the following $\mathrm{X}$-ray pictures :

5/11/27. X-ray shows upper right chest almost clear, but the right base remains opaque. The trachea is seen well to the right side, but the apex of the heart can be noted to the left of the spine, showing that it is returning to its normal fosition; the right korder of the mediastinal shadow can be seen (Fig. 3).

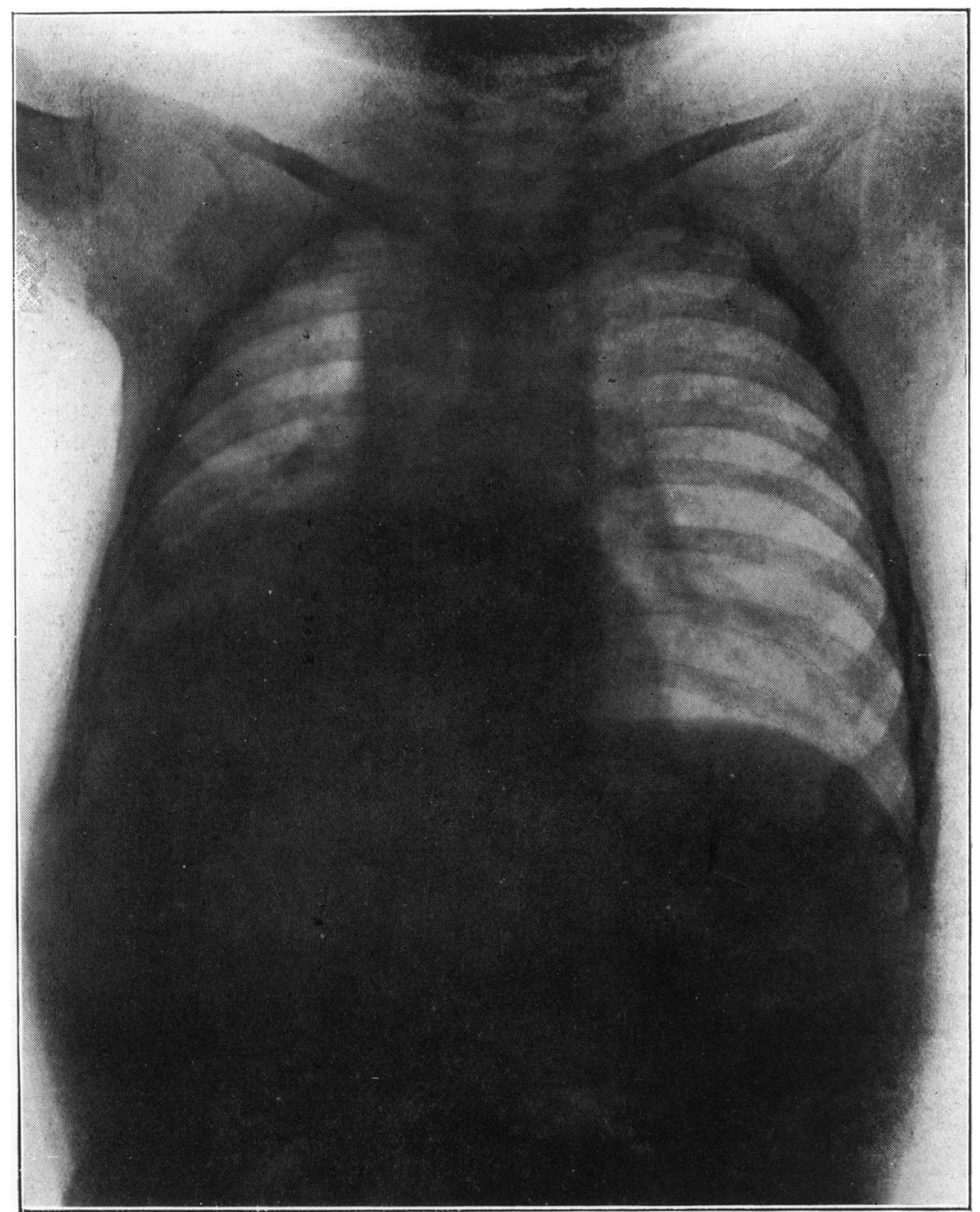

Fig. 3. Case III., L.M. 5/11/27. The right upper chest is almost clear; the apex of the heart can be seen appearing to the left of the spine, but there is still marked cardiac displacement towards the side of the lesion. 
12\%11/27. X-ray still shows area of density at right lower lobe, probably a patch of unre. solved pneumonic consolidation. The heart is returning to the normal position.

1/12/27. X-ray now shows unresolved area much improved.

17/12/27. Physical examination shows no physical signs whatever, and the heart appears normal in position. X-ray shows the heart and trachea to be still displaced and the unresolving area to have increased in size (Fig. 4).

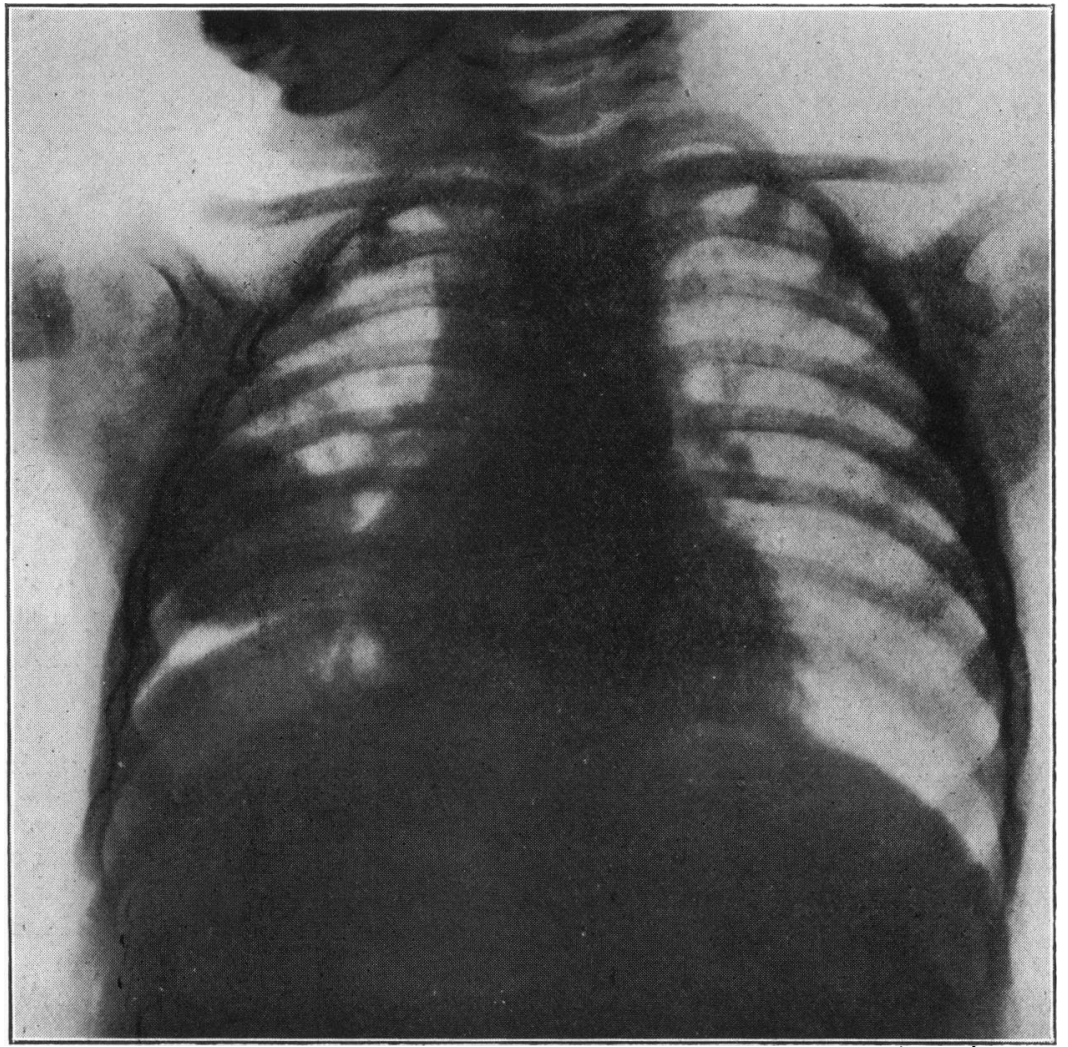

Fig. 4. Case III., L.M. 17/12/27. The area of unresolved pneumonia at the right base is seen. The heart is gradually returning to its normal position, although it is still well to the right side.

Eight days after discharge from hospital to the out-patient department, the child had a relapse which was marked with cyanosis, dyspnœa, a croupy cough and much frothy expectora tion. Owing to lack of accommodation, arrangements were made for his admission to the Eas $t$ London Hospital for Children, where he was placed under the care of Dr. G. Bourne, by whose courtesy we were subsequently permitted to examine the child, and who supplied us with the following notes of the case with permission to report them.

$28 / 12 / 27$. The percussion note at the right base is impaired: bronchial breath sounds heard at this area and moist sounds throughout both lungs. The heart sounds are bes $t$ heard to the right side of sternum. The area of cardiac dullness cannot be defined.

$31 / 12 / 27$. The dullness and bronchial breath sounds are spreading throughout the whole of the right lung. Moist sounds heard at the left lower lobe.

3/1/28. Laryngeal stridor very marked : apparent collapse of whole of right lung and marked cyanosis. 
6/1/28. Child much the same except that the whole of left lung is full of moist sounds. Child died that evening.

Post-mortem Examination. Heart was found to be lying almost entirely to the right of middle line. There was a small effusion (? terminal) in the pericardial sac. The left lung was large, full of air, with some purulent bronchitis at the base and a small abscess at the apex of the lower lobe. Patch of pleurisy over left lower lobe. There was consolidation of the middle and upper lobes of the right lung with complete collapse of the right lower lobe. The trachea was normal.

Case IV. L.P., age 6 years. Four days ago woke up screaming and delirious. For two days remained delirious, and was feverish. Had attacks of 'screaming with apparent pain in right side. Vomited twice on day of admission.

22/5/28. Child is drowsy but not delirious. Looks excessively ill and has a high temperature and rapid respiration.

No physical signs, but irritability, head retraction and meningism.

$23 / 5 / 28$. Impaired percussion note right apex. Breathing high pitched. No crepitations heard. Apex beat 4th space half-inch internal to nipple line, sounds normal. Tonsils unhealthy. Naso-pharyngeal discharge. No other physical signs, and head retraction is now absent. Cerebro-spinal fluid normal. Wassermann reaction negative.

$\mathrm{X}$-ray shows an increase in density of right upper lobe, with displacement of heart to right.

31/5/28. No high pitched breath sounds, but some impairment of percussion note right upper lobe, and on deep breathing and coughing crepitations are heard at bases, and also at right lower lobe. Looks better. Temperature and respiration settling to normal. Area of cardiac dullness does not extend to right, but apex beat still internal to nipple.

4/6/28. X-ray shows the whole of the right chest to be less translucent than the left. The upper part of the right upper lobe appears clearer; the lower part is mottled. The right lower lobe seems slightly involved now. The left chest is clear. The heart and trachea are still to the right, their position being similar to that noted on $24 / 5 / 28$.

7/6/28. Chest seems clear now. Heart normal in position.

11/6/28. The temperature and respiration rate have been normal for two weeks and the child seems quite well.

Case V. J.R., age 10 months. There is a strong family history of tuberculosis on the father's side. 11 days ago child was taken ill and diagnosed as having pneumonia, by private practitioner. For the past week he has had a high temperature with cough, and has been vomiting frequently.

26/9/28. High pitched breath sounds noted over the left lower lobe, but no other definite physical signs. Heart appears normal in position.

$6 / 10 / 28$. The respiration rate is raised and the temperature high and swinging. The breath sounds at the left base are diminished, otherwise nothing is to be noted. On account of the swinging temperature left lung explored by needle. No definite pus obtained. A catheter specimen of urine, acid in reaction, contained a trace of albumin, a few leucocytes and epithelial cells; from it B. coli and proteus cultured. Von Pirquet reaction negative. Stomach washings fail to reveal tubercle bacilli.

16/10/28. X-ray shows density over left base with heart and trachea slightly to right. Left diaphragm does not show up under screen. Slightly increased density right lower lote (Fig. 5). As a result of the X-ray left base was again needled with a negative result. The temperature and respiration rate are now normal.

28/10/28. The child, who has been discharged, has attended as an out-patient, and physical examination shows diminished breath sounds both right and left bases, with hyper-resonance at right upper lobe and heart displaced markedly to the right.

29/10/28. X-ray now shows wedge-shaped area of consolidation at right base, left base being clear. There is no evidence of pneumothorax. The heart is well over to the right (Fig. 6). 
31/10/28. Cough is marked, temperature raised. Physical examination shows left base clear. Hyper-resonance left axillary region and over præcordial area. Impaired percussion note and diminished breath sounds at right base. Area of cardiac dullness not defined owing to hyper-resonance over præcordium (emphysema). Apex beat felt and heard loudest just to left edge of sternum. Child re-admitted to hospital.

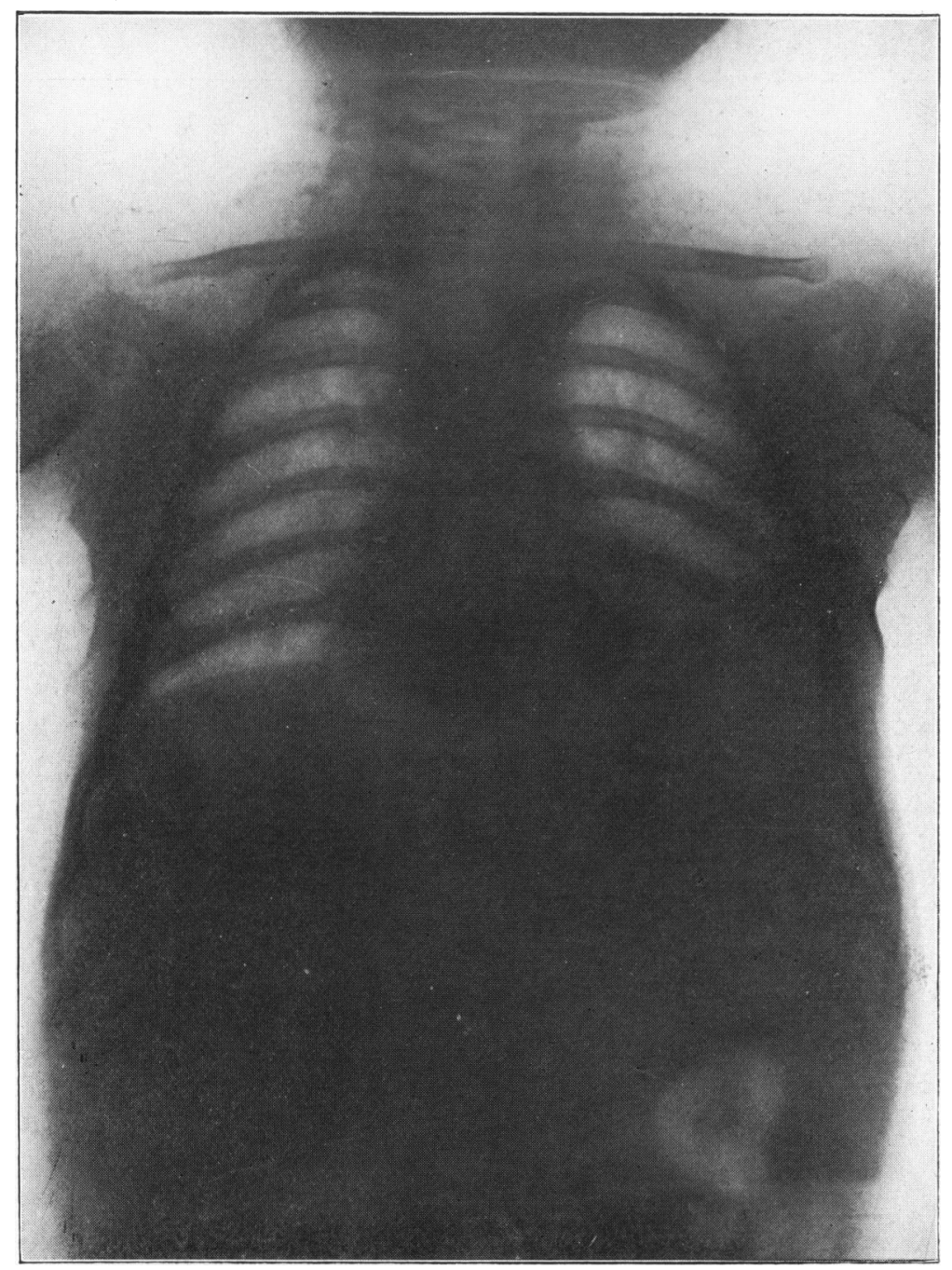

Fig. 5. Case V., J.R. 15/10/28. The heart and trachea are slightly displaced to the right side, and there is slight increase in density at the right lower lobe. The left lower chest is dull and a diagnosis of fluid at the left base was made, but no fluid was obtained on needling.

5/11/28. Temperature and respiration, which have both been raised, now fallen to normal. 9/11/28. Temperature remains steady and normal. Child seems well. Impaired percussion note at right base, otherwise no physical signs in lungs. Heart going back to normal position, the apex beat now being half-inch internal to nipple line. 
20/2/29. Since last seen child has been ill with an exacerbation of the previous condition For two months has been receiving artificial sunlight treatment, and now looks in good health, and is said to be very well. No physical signs in lungs. Apex beat of heart $\frac{1}{4}$-inch internal to nipple line.

$\mathrm{X}$-ray shows the heart normal in size, shape, and position; the trachea is slightly to the right side, and there is some slight increase of density at the lower part of the right upper lobe which is probably largely due to inter-lobar thickening. This would explain the fact that the trachea is not in the mid-line.

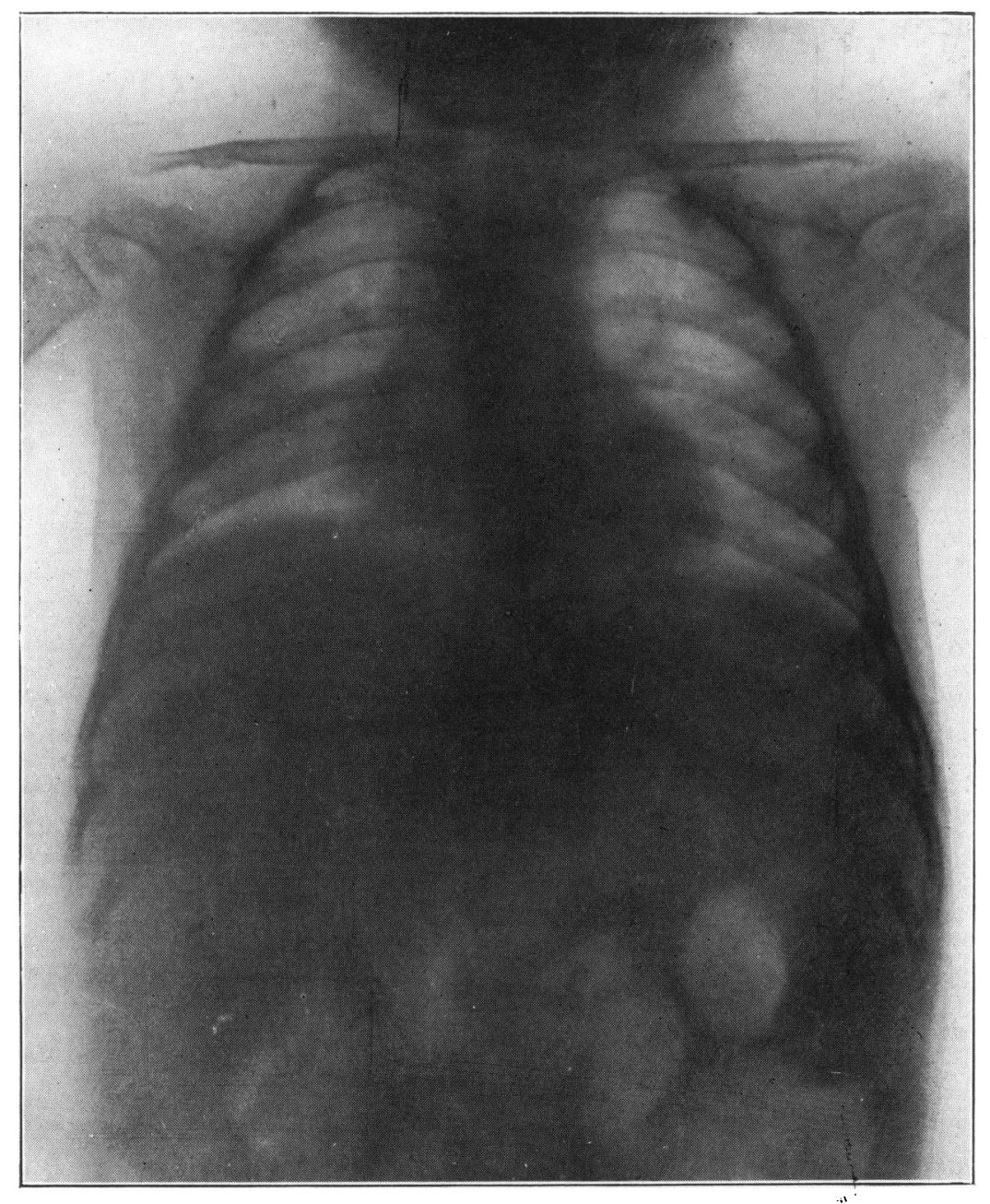

FIG 6. Case V., J.R. 29/10/28. The left base is now clear, and no pneumo-thorax is seen. The heart is still well displaced to the right. There is a local area of pneumonia at the right lower lobe.

\section{Discussion.}

It is by no means easy in every case of pneumonia to make a clear cut diagnosis of its type, whether it be lobar or broncho-pneumonia. Somerville ${ }^{6}$ in a rccent paper shows that by far the greater number of cases of pneumonia, 
occurring in children under 6 years of age are, on a basis of pathological examinations carried out post-mortem, broncho-pneumonic in type. Certainly this is the case in infants. This observer thinks that pathological examination is the only true criterion of the type of disease that has occurred. As criteria of the type of pneumonia present in our cases, the history, physical signs, and course of the disease have all been taken into account rather than the physical signs alone. Considered thus, three of our cases appear to be bronchopneumonic in type (Cases I, II and III) and two (Cases IV and V) appear to be cases of true lobar pneumonia.

In Case $V$ first the left and subsequently the right side was affected. During the first attack, the heart did not appear to deviate towards the affected side, but when the right lung was affected, the heart showed definite displacement. In four of the cases (Cases I, II, III and IV) the right lung was chiefly affected, the heart deviating towards that side.

Definite emphysema was noted on the side unaffected by pneumonia in two cases (Cases II and V), and in one case where the right side gave evidence of pneumonia, the area of cardiac dullness could not be defined by percussion (Case I). The X-ray photographs reproduced are those which best exemplify the conditions under discussion.

\section{Summary and Conclesions.}

Five cases are described in which during an attack of pneumonia, the heart deviated towards the side of the lesion, subsequently returning slowly to a normal position as recovery occurred.

A consideration of these cases and of other similar cases reported in the literature, points to the fact that this phenomenon is not infrequent inchildhood, and would in all probability be more often noted if specially looked for by physical examination, and if radiograms of the chest were taken early. Displacement of the heart appears to be brought about by the traction exercised by shrinkage due to partial collapse of the affected lung, aided by the push of the sound lung which is frequently distended by compensatory emphysema.

This cardiac displacement is not caused by a pulling over due to fibrosis, since it occurs in the acute stage of the disease, and the heart subsequently returns to its normal position. Moreover, neither by physical nor by radiological examination can evidence of fibrosis be noted.

We wish to thank Dr. Maitland-Jones for permission to report these cases, which were admitted to the Hospital under his care.

\section{REFERENC'ES.}

1. Maitland-Jones, A. G., Proc. Roy. Soc. Med. (Sect. Study of Dis. ('hild.), Lond., 1928, XXI, 1327.

2. Thoenes, F., Monutschr.f. Kinderh., Leipsic, 1922, XXIII, 353.

3. Wallgren, A., Acta Pad., Uppsula, 1923-24, III, 81.

4. Griffith, J. P. ('., Am. J. Med. Sc., Phil., 1927, ('LXXIV, 448.

5. Coryllos, P. M., \& Birnbaum, G. L., Arch. Surg., ('hicago, 1929, XVIII, 190.

6. Somerville, O., Arch. Dis. Childhood, Lond., 1928, III, 194. 